\title{
The I/LWEQ Domain in RapGAP3 Required for Posterior Localization in Migrating Cells
}

\author{
Mi-Rae Lee, Hyeseon Kim, and Taeck J. Jeon*
}

Cell migration requires a defined cell polarity which is formed by diverse cytoskeletal components differentially localized to the poles of cells to extracellular signals. RapGAP3 transiently and rapidly translocates to the cell cortex in response to chemoattractant stimulation and localizes to the leading edge of migrating cells. Here, we examined localization of truncated RapGAP3 proteins and found that the I/LWEQ domain in the central region of RapGAP3 was sufficient for posterior localization in migrating cells, as opposed to leading-edge localization of full-length RapGAP3. All truncated proteins accumulated at the leading edge of migrating cells exhibited clear translocation to the cell cortex in response to stimulation, whereas proteins localized to the posterior in migrating cells displayed no translocation to the cortex. The I/LWEQ domain appears to passively accumulate at the posterior region in migrating cells due to exclusion from the extended front region in response to chemoattractant stimulation rather than actively being localized to the back of cells. Our results suggest that posterior localization of the I/LWEQ domain of RapGAP3 is likely related to F-actin, which has probably different properties compared to newly formed F-actin at the leading edge of migrating cells, at the lateral and posterior regions of the cell.

\section{INTRODUCTION}

Directional cell movement in response to chemoattractant stimulation requires a defined cell polarity in which cytoskeletal components are differentially localized to both poles of cells. At the front of cells, F-actin is polymerized and leads to protrusions of the membrane surface, whereas in a coordinated manner, myosin II assembly occurs at the posterior of cells and mediates contraction. How these asymmetries are spatially organized and maintained is one of the central questions in under-

Department of Biology and Brain Korea 21- Plus Research Team for Bioactive Control Technology, College of Natural Sciences, Chosun University, Gwangju 501-759, Korea

${ }^{*}$ Correspondence: tjeon@ chosun.ac.kr

Received 29 October, 2013; revised 29 November, 2013; accepted 9 December, 2013; published online 6 March, 2014

Keywords: actin cytoskeleton, cell migration, Dictyostelium, I/LWEQ domain, RapGAP standing cell migration (Lee and Jeon, 2012; Ridley et al., 2003; Sanchez-Madrid and Serrador, 2009).

Cell polarity is formed by a series of signaling molecules, which are differentially activated upon ligand binding to surface receptors. One of the early responses to chemoattractant stimulation is the activation of Ras proteins. Activated Ras proteins are enriched at the leading edge of chemotaxing cells, where they locally activate signaling molecules (Lee and Jeon, 2012; Ridley et al., 2003). Phosphatidylinositol 3-kinases (PI3Ks) rapidly accumulate at the leading edge of cells in response to a chemoattractant, whereas PTEN becomes restricted to the sides and the rear, although it is not yet clear what regulates the localization of PI3Ks and PTEN. The reciprocal localization and activation of PI3K and PTEN lead to accumulation of phosphatidylinositol $(3,4,5)$ trispohsphate (PIP3) at the leading edge, which helps guide the local polymerization of F-actin as well as pseudopod extension at the leading edge of cells possibly by recruiting pleckstrin homology $(\mathrm{PH})$ domain-containing proteins such as PhdA, CRAC, and PKB (Kortholt and van Haastert, 2008; Ridley et al., 2003). Assembled myosin II is preferentially found in the rear body and along the lateral sides in a decreasing posterior-to-anterior gradient where it is involved in uropod retraction. Assembly of myosin II at the back of cells is mainly regulated by several signaling molecules, including PAKa, the cGMP signaling pathway, and the Rap1 signaling pathway (Kortholt and van Haastert, 2008; Lee and Jeon, 2012).

The small GTPase Rap1 is involved in the control of diverse cellular processes, including integrin-mediated cell adhesion, cadherin-based cell-cell adhesions, and cell polarity in mammalian cells as well as cell adhesion, phagocytosis, and cell migration in Dictyostelium (Kooistra et al., 2007; Kortholt et al., 2010; Raaijmakers and Bos, 2009). Rap1 is rapidly and transiently activated at the leading edge of cells during cell migration in response to chemoattractant stimulation. Leading-edge activation of Rap1 regulates cell adhesion and helps establish cell polarity by locally modulating myosin II assembly and disassembly through the Rap1/Phg2 signaling pathway (Cha et al., 2010; Jeon et al., 2007a; Kortholt and van Haastert, 2008; Lee and Jeon, 2012; Mun and Jeon, 2012). Recent reports have demonstrated that spatial and temporal regulation of Rap1 activity by Rap1 GAP proteins is required for proper cell migration. RapGAP1 was identified as a specific GAP protein for Rap1 and is involved in the regulation of Rap1 activity in the anterior of chemotaxing cells to control cell-substratum adhesion and myosin II assembly during chemotaxis (Jeon et al., 
2007b). RapGAPB and RapGAP3 are required for the correct sorting behavior of different cell types during development (Jeon et al., 2009; Parkinson et al., 2009). RapGAP3 mediates deactivation of Rap1 at the late mound stage of development and plays an important role in regulating cell sorting during apical tip formation, when the anterior-posterior axis of the organism is formed, by controlling cell-cell adhesion and cell migration. RapGAP3 transiently and rapidly translocates to the cell cortex in response to chemoattractant stimulation, which is dependent on F-actin polymerization, and localizes to the leading edge of migrating cells (Jeon et al., 2009; Lee and Jeon, 2012).

To understand the spatial mechanism by which directs localization of RapGAP3 during migration, we examined the subcellular localization of truncated RapGAP3 proteins and found that the I/LWEQ domain in the central region of RapGAP3 is required for posterior localization of the protein during cell migration. Here, we present an analysis of the RapGAP3 fragments required for polarized localization of the protein in migrating cells.

\section{MATERIALS AND METHODS}

\section{Strains and plasmids}

Dictyostelium wild-type KAx-3 cells were cultured axenically in $\mathrm{HL} 5$ medium at $22^{\circ} \mathrm{C}$. The myosin II, gCA/sGC, and gbp a/b null strains were obtained from the DictyBase Stock Center. The expression plasmids for GFP-coronin and RFP-coronin were described previously (Cha and Jeon, 2011; Jeon et al., 2007b). For expression of GFP-RapGAP3, the full coding sequence of rapGAP3 cDNA was generated by RT-PCR, cloned into the Bgll-Xhol site of pBluescript KS (-), sequenced, and subcloned into the expression vector pEXP-4(+) containing a GFP fragment (Jeon et al., 2009). For expression of the truncated RapGAP3 proteins shown in Fig. 1A, the regions in RapGAP3 marked in the diagram (Fig. 1A) were amplified by PCR and cloned into the Bgll-Xhol site of a pEXP-4 vector containing a GFP fragment. The plasmids were transformed into KAx-3 cells, and the cells were maintained in $20 \mu \mathrm{g} / \mathrm{ml}$ of $\mathrm{G} 418,50 \mu \mathrm{g} / \mathrm{ml}$ of hygromycin, or both as required.

\section{Chemotaxis and image acquisition}

The subcellular localization of proteins in response to chemoattractant stimulation was examined as described previously (Cha and Jeon, 2011; Jeon et al., 2007b). Vegetative cells were washed twice with $\mathrm{Na} / \mathrm{K}$ phosphate buffer, resuspended at a density of $5 \times 10^{6}$ cells $/ \mathrm{ml}$ in Na/K phosphate buffer, and pulsed with $30 \mathrm{nM}$ cAMP at 6 -min intervals for $5 \mathrm{~h}$. The pulsed cells were placed on glass-bottomed microwell plates. For imaging chemotaxing cells, a micropipette filled with $150 \mu \mathrm{M}$ cAMP was positioned near the cells for stimulation. Images of chemotaxing cells were taken at time-lapse intervals of $6 \mathrm{~s}$ for 30 min using an inverted microscope (IX71; Olympus, Japan) with a camera (DS-Fi1; Nikon, Japan).

Quantitative analysis of membrane or cortical localization of GFP fusion proteins was performed as described previously (Jeon et al., 2007b; Sasaki et al., 2004). Aggregation competent cells were allowed to adhere to the plate for $10 \mathrm{~min}$. Cells were then uniformly stimulated with cAMP by quickly pipetting $250 \mu$ of $150 \mu \mathrm{M}$ cAMP into the plate containing cells. Fluorescence images were taken at time-lapse intervals of $1 \mathrm{sec}$ for 1 min using an inverted microscope. The frames were captured using NIS-elements software (Nikon) and analyzed using ImageJ software (National Institutes of Health, USA). The intensity of fluorescence at the cell cortex was measured, and the level of cortical GFP was calculated by dividing the intensity at each time point $(\mathrm{Et})$ by the intensity before stimulation (Eo).

\section{RESULTS}

\section{Subcellular localization of truncated RapGAP3 fragments} in migrating cells

RapGAP3 has Rap1-specific activity and plays an important role in the process of development by regulating cell-cell adhesion in multicellular organisms. RapGAP3 is recruited to the cell cortex transiently and rapidly in response to chemoattractant stimulation and localizes to the leading edge of migrating cells (Jeon et al., 2009). To further understand the roles of RapGAP3 in the regulation of Rap1 activity during migration and development in Dictyostelium, we further investigated the roles of RapGAP3 protein domains in regulating the localization of RapGAP3 during migration.

RapGAP3 contains three $\mathrm{PH}$ domains at the $\mathrm{N}$-terminal as well as a GAP domain at the C-terminal region (Fig. 1A). In addition, another domain similar to the I/LWEQ domain, known as the F-actin binding domain and originally characterized in the analysis of Talin proteins (Brett et al., 2006; McCann and Craig, 1997), was found in the central region of RapGAP3 when the amino acid sequence of the central region of RapGAP3 was compared with those of TalinA and TalinB using a sequence analysis tool (block maker).

To investigate the role of each domain in RapGAP3 function during the developmental process and movement, we prepared a series of truncated fragments fused with GFP and analyzed localization of the fragments during chemotaxis moving up a gradient of CAMP chemoattractants (Fig. 1B). Full-length RapGAP3 localized to the leading edge of migrating cells as previously reported (Jeon et al., 2009). Surprisingly, removing the GAP domain from RapGAP3 resulted in reverse localization of the proteins from the front to the back of cells (Fig. 1B). Cells expressing full-length RapGAP3 (\#624) displayed high accumulation of the proteins at the leading edge along with a small amount at the rear and sides of cells. In contrast, cells expressing the fragments without the GAP domain (\#628 and \#627) exhibited disappearance of the protein at the leading edge and accumulation with a decreasing gradient from the rear to the front of cells. GFP-fusion protein containing the GAP domain alone was found in the cytosol. These results suggest that RapGAP3 contains all of the properties required for localization to each pole in cells, whereas the GAP domain plays a role in localizing proteins to the anterior of cells during migration but is not sufficient for anterior localization.

To characterize the region required for posterior localization of the proteins, we further examined the localization of the fragments truncated at the $\mathrm{N}$-terminus. The $\mathrm{PH}$ domain alone (\#626) was enriched at the leading edge. Cells expressing the $\mathrm{PH}$ domain-truncated protein (\#636) showed high enrichment at the leading edge as well as slight accumulation at the posterior and sides of cells, similar to that of cells expressing fulllength RapGAP3. This result suggests that the $\mathrm{PH}$ domain functions in anterior localization of the protein but not required for localization to the front. It appears that there is an additional region in RapGAP3, possibly the GAP domain since GAP domain-deleted fragments were not found in the anterior region, functions in anterior localization during cell migration. Interestingly, the I/LWEQ domain without the $\mathrm{PH}$ or GAP domain (\#137) was found at the posterior of migrating cells, similar to GAP domain-truncated proteins, indicating that the I/LWEQ 
A

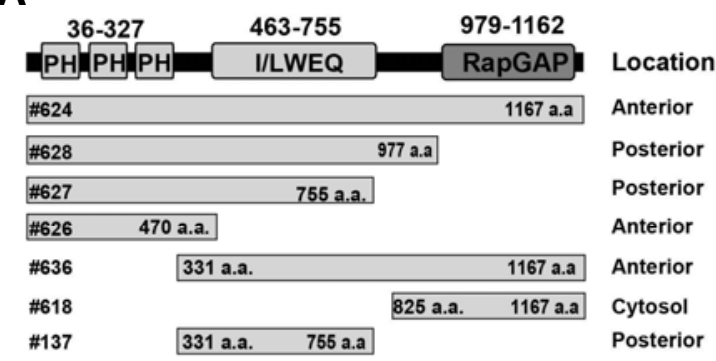

B

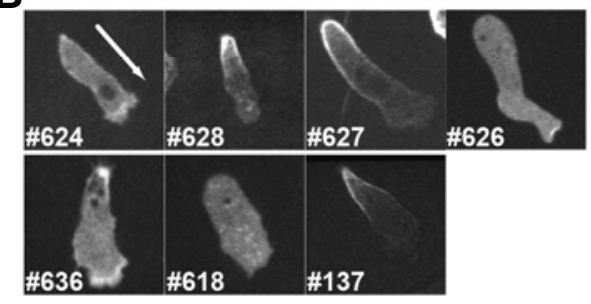

D

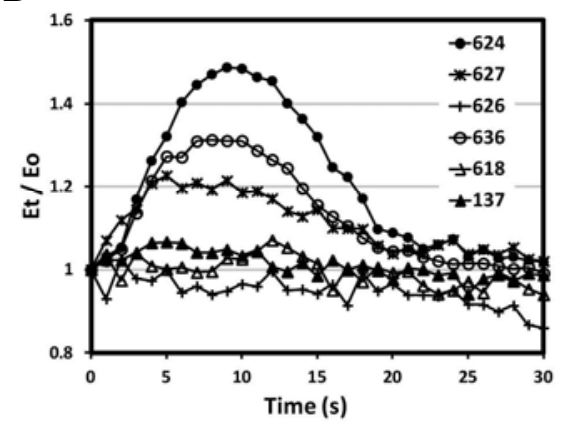

C

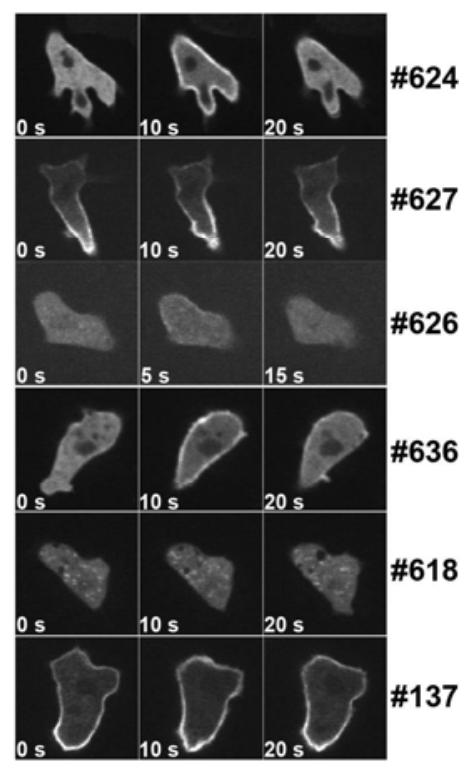

Fig. 1. Localization of truncated RapGAP3 proteins. (A) Schematic diagram of truncated RapGAP3 proteins. The locations of the proteins during chemotaxis are described at the end of each fragment. (B) Localization of the fragments in chemotaxing cells. The arrow indicates the direction of movement. (C) Translocation to the cell cortex in response to chemoattractant stimulation. Cells expressing truncated RapGAP3 proteins were uniformly stimulated with cAMP, after which the images were recorded. Representative images at 0,10 , and $20 \mathrm{~s}$ are presented. (D) Translocation kinetics of the fragments of RapGAP3 to the cell cortex from time-lapse recordings. Fluorescence intensity at the cell cortex was quantitated as described previously (Jeon et al., 2007a). Graphs are the means of several cells from videos from at least three separate experiments. domain alone is sufficient for posterior localization. In conclusion, our results demonstrate that three regions, the $\mathrm{PH}, \mathrm{I} / \mathrm{LWEQ}$, and GAP domains, contribute to the localization of RapGAP3 during migration. Further, the $\mathrm{PH}$ and GAP domains play roles in the anterior localization of RapGAP3, whereas the I/LWEQ domain is sufficient for posterior localization. The leading-edge localization of RapGAP3 suggests that the I/LWEQ domain in the full-length RapGAP3 might be masked by other domains and is unlikely to interact with the binding partners.

\section{Translocation to the cell cortex in response to} chemoattractant stimulation

It has been reported that RapGAP3 transiently and rapidly translocates to the cell cortex in response to chemoattractant stimulation with a peak of around $10 \mathrm{~s}$ (Jeon et al., 2009). We examined the translocation of truncated fragments of RapGAP3 to the cell cortex and analyzed translocation kinetics to further understand the factors affecting localization of RapGAP3. While full-length RapGAP3 (\#624) showed transient translocation to the cell cortex with a peak at around $10 \mathrm{~s}$ after stimulation, the translocation of GAP domain-deleted RapGAP3 (\#627 and \#628 data not shown) to the cell cortex was not obvious (Figs. 1C and 1D). This result indicates that the GAP domain is required for translocation to the cell cortex in response to chemoattractant stimulation. The $\mathrm{PH}$ domain alone (\#626), which was previously reported to transiently translocate to the cell cortex, exhibited translocation to the cell cortex upon chemoattractant stimulation, and $\mathrm{PH}$ domain-deleted fragments containing the GAP domain also showed clear translocation to the cortex. The I/LWEQ domain alone (\#137) showed no clear translocation upon uniform stimulation, as GAP domain-deleted proteins. These results suggest that the $\mathrm{PH}$ and GAP domains are involved in the translocation of RapGAP3 to the cell cortex in response to chemoattractant stimulation, and the GAP domain has a main function in translocation since deletion of the GAP domain but not $\mathrm{PH}$ domain prevented translocation of the proteins.

More importantly, translocation of the proteins to the cortex upon uniform chemoattractant stimulation is likely to be correlated to the spatial localization of the proteins in migrating cells. All proteins accumulated at the leading edge of migrating cells (\#624, \#626, and \#636) exhibited clear translocation to the cell cortex in response to uniform chemoattractant stimulation, whereas proteins localized to the posterior in migrating cells (\#628, \#627, \#137) displayed no translocation to the cortex. Leadingedge localization appears to be correlated to the transient accumulation of proteins by chemoattractant stimulation. These results suggest that leading-edge accumulation of the proteins might be actively induced in response to chemoattractant stimulation, whereas posterior localization of proteins might be passively localized by binding to preexisting cytoskeletal components and excluded from the anterior by actively induced proteins. 
A

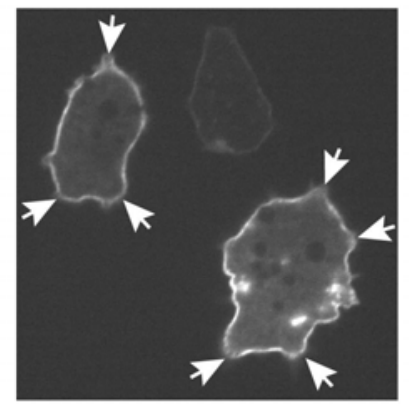

D

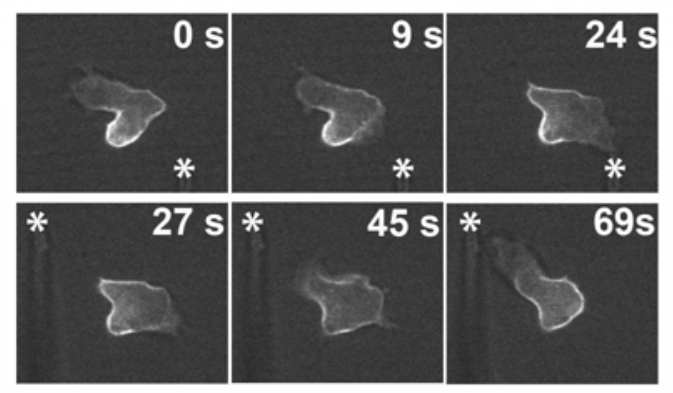

C
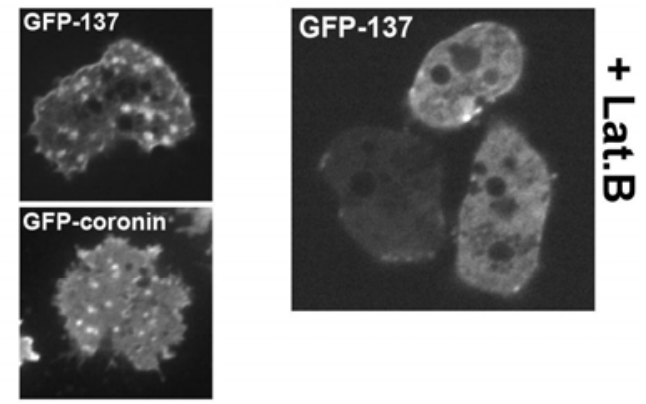

E

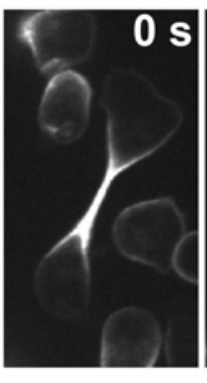

Fig. 2. Analysis of fragment containing the I/LWEQ domain. Cells expressing GFP-137 plasmid, which contains the I/LWEQ domain, were analyzed. (A) Localization of the I/LWEQ domain in vegetative cells. The arrowheads indicate the protruding regions. (B) Actin foci at the bottom of cells expressing the I/LWEQ domain (GFP-137) or GFPcoronin, which is a marker protein for newly formed F-actin. (C) Localization of the $I / L W E Q$ domain in the presence of LatA, which is an inhibitor of $F$-actin assembly. (D) Localization of the I/LWEQ domain in chemotaxing cells upon changing the position of the micropipette filled with the chemoattractant CAMP. The asterisk indicates the location of the micropipette. (E) Localization of the $\mathrm{I} / \mathrm{LWEQ}$ domain during cytokinesis.

\section{Localization of the I/LWEQ domain}

The I/LWEQ domain was originally identified on the basis of sequence similarity in several F-actin binding proteins and has been shown to mediate cytoskeletal localization and F-actin binding (Brett et al., 2006; McCann and Craig, 1997; Senetar et al., 2004). Even though the I/LWEQ domain in RapGAP3 is expected to differentially accumulate at the front of migrating cells, in our previous localization study, the I/LWEQ domain of RapGAP3 was found at the rear and was shown to be sufficient for posterior localization. To further understand the mechanism behind the asymmetric distribution of the protein during migration, we examined localization of the I/LWEQ domain of RapGAP3 in more detail (referred to Localization domain, LD).

In vegetatively growing cells, the LD was localized at the cell cortex. Interestingly, fluorescence of this protein decreased at protruding regions (Fig. 2A, indicated by arrows), where it is known that $\mathrm{F}$-actin is polymerized and $\mathrm{F}$-actin binding proteins usually accumulate (Kolsch et al., 2008; Ridley et al., 2003). This result is unexpectedly contrary to the expectation that the I/LWEQ domain might bind to F-actin and become localized to the front of migrating cells. It seems that the LD was excluded by the newly formed F-actin at protruding regions rather than binding to F-actin. However, controversial data in line with the notion that the $\mathrm{I} / \mathrm{LWEQ}$ domain is excluded from F-actin at protruding regions were obtained from the following experiments. It is known that cells form actin foci at their bottoms, which are exhibited by GFP-coronin, a marker protein for newly formed Factin (Fig. 2B) (Cha and Jeon, 2011; Jeon et al., 2007b). GFPLD was found at foci at the bottom of cells, similar to the actin foci shown by GFP-coronin. In addition, in the presence of Latrunculin $A$, an inhibitor of $F$-actin polymerization, most GFP-LD dissociated from the cell cortex, suggesting that the $\mathrm{I} / \mathrm{LWEQ}$ domain localizes to the cell cortex in an F-actin dependent manner (Fig. 2C). The controversial result that the I/LWEQ domain seems to be excluded from the protruding region by newly formed F-actin along with the occurrence of F-actin dependent localization to the cell cortex and actin foci at the bottom of cells raises the possibility of two more types of F-actin with different properties.

In moving cells, dynamic localization of GFP-LD was investigated by changing the location of the pipette filled with chemoattractants (Fig. 2D). The pipette filled with chemoattractants was placed at a position displaying a high level of GFP-LD near the cells $(0 \mathrm{sec})$. Shortly after positioning of the pipette, the cells produced protrusions toward the pipette while, at the same time, the intensity of the fluorescence decreased at protruding regions ( $9 \mathrm{~s}$ and $24 \mathrm{~s}$ ). It seems that the amount of GFP-LD at the cortex was rapidly diluted upon extension of the cell cortex toward the pipette, after which the proteins close to the pipette almost disappeared while the other side of cells showed reverse accumulation of GFP-LD. When the pipette was changed to another position ( $27 \mathrm{~s}$ ), a high level of GFP-LD at the cell cortex close to the pipette (front of moving cells) disappeared as previously shown. It appears that GFP-LD passively accumulated at the posterior region in migrating cells due to exclusion from the extended front region in response to chemoattractant stimulation rather than actively being localized to the back of cells. It is known that F-actin accumulates at each pole of dividing cells, whereas myosin localizes to the cleavage furrow (Lee and Jeon, 2012; Ridley et al., 2003). The I/LWEQ domain was found at the cleavage furrow as well (Fig. 2E).

\section{Colocalization of the I/LWEQ domain with F-actin cytoskeleton}

To gain insights into the relationship between the I/LWEQ domain and F-actin, we expressed both GFP-LD and RFP. coronin, which is a marker protein for newly formed F-actin, and then examined their dynamic localization in moving cells and translocation kinetics to the cell cortex in response to chemoattractant stimulation simultaneously. Cells expressing GFP-LD 
A

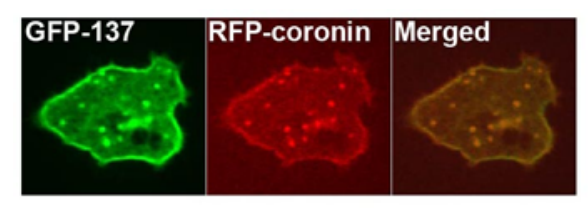

B

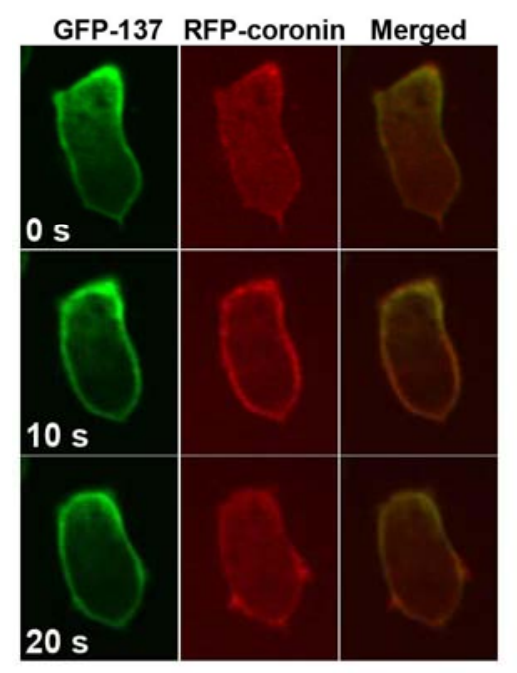

C

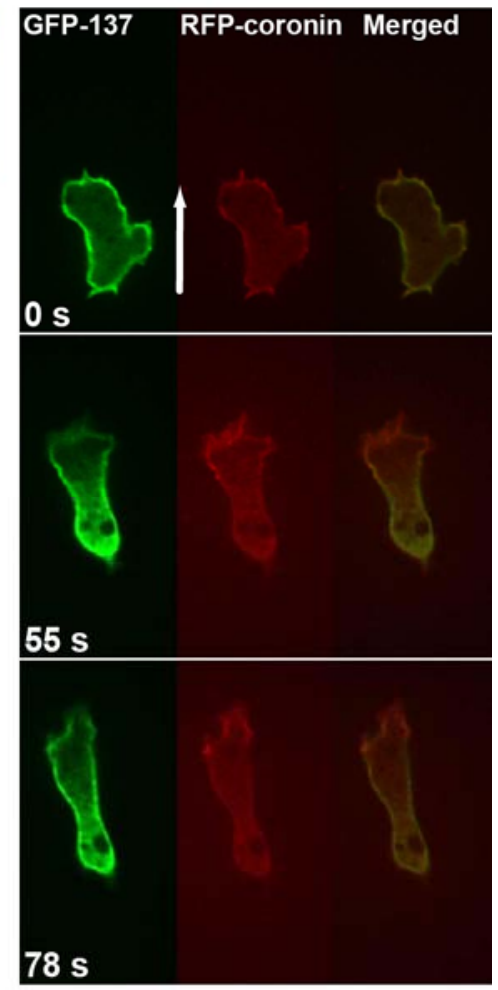

Fig. 3. Colocalization of the I/LWEQ domain with coronin. Dual-view analyses of cells expressing both GFP-137 (the I/LWEQ domain) and RFP-coronin, which is a marker protein for newly formed F-actin. (A) Bottom sections of cells. (B) Translocation of both proteins to the cell cortex in response to chemoattractant stimulation was imaged. (C) Localization of both proteins during chemotaxis. The arrow indicates the direction of the cell. showed foci at the bottom of cells in the previous experiment. To determine if foci found in cells expressing GFP-LD are actin foci or colocalize with those exhibited in cells expressing RFP. coronin, we examined the bottom of cells expressing both GFP. LD and RFP-coronin. As expected, RFP-coronin as well as GFP-LD were found at actin foci at the bottom of cells, and the locations of GFP-LD and RFP-coronin appear to exactly match in the merged images (Fig. 3A). This indicates that the foci shown by GFP-LD are localized to actin foci, and GFP-LD and RFP-coronin colocalize at least at the bottom of cells.

Next, we compared the translocation kinetics of both GFP-LD and RFP-coronin to the cortex (Fig. 3B). RFP-coronin exhibited transient translocation to the cell cortex, implying that F-actin newly and transiently formed at the cortex in response to chemoattractant stimulation, but no such translocation to the cortex was observed for GFP-LD. Compared to RFP-coronin, a high level of GFP-LD was found in unstimulated cells, and this high level was continually present at the cortex without alteration of fluorescence intensity upon chemoattractant stimulation. The merged images clearly show the differences in protein levels at the cell cortex. These results suggest that the localization of GFP-LD at the cell cortex is not related to newly formed F-actin.

To understand spatial localization of the two proteins, we examined the locations of the proteins in migrating cells (Fig. 3C). In not-yet polarized cells, the two proteins GFP-LD and RFPcoronin were found at partially overlapped regions at the cortex. A small difference in their localization was found at the protruding regions of non-moving cells. RFP-coronin accumulated at protruding regions, indicating F-actin was newly assembled, whereas GFP-LD was present at a relatively low level compared with other cell regions. In moving cells, RFP-coronin diffe- rentially localized to the leading edge, in which F-actin is mainly polymerized. In contrast, the GFP-LD level was lower at the front of cells close to the pipette filled with chemoattractants, resulting in a decreasing localization gradient from the posterior to the front of cells. These results suggest that GFP-LD might be excluded from the leading edge of migrating cells by newly formed F-actin.

\section{Localization of the I/LWEQ domain in mutants}

To determine which signaling pathways are involved in the posterior localization of LD in moving cells, we introduced GFPLD into myoll, $g C A / s G C$, and $g b p$ a/b null cells and examined localization of the protein in mutants (Fig. 4). As in wild-type cells, GFP-LD was localized to the posterior region in all mutant cells, suggesting that Myosin II and the cGMP pathway are not involved in localization of GFP-LD in the posterior of cells during migration. In the presence of LY, which is an inhibitor of PI3Ks, GFP-LD exhibited normal posterior localization as in control cells, suggesting that localization of GFP-LD is independent of the PI3K pathway.

\section{DISCUSSION}

In this study, we investigated the localization of RapGAP3 fragments in migrating cells and found that the I/LWEQ domain in the central region of RapGAP3, which is known as an actinbinding region and originally identified by comparison with Talin proteins (Brett et al., 2006; McCann and Craig, 1997; Senetar et al., 2004), localized to the posterior, even though full-length RapGAP3 was found at the anterior of cells during migration. The GAP domain was required but not sufficient for localization 

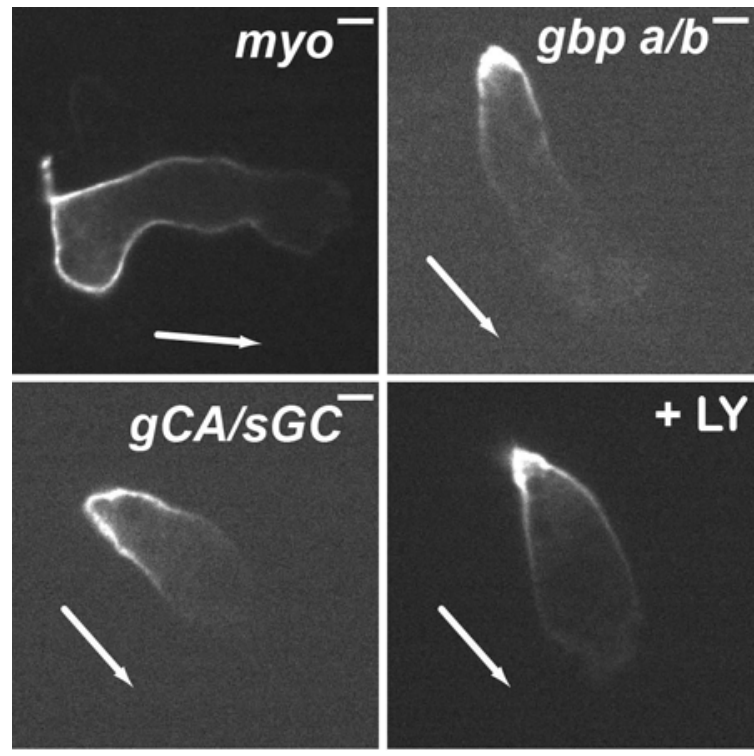

to the anterior of cells and transient translocation to the cell cortex in response to chemoattractant stimulation. Cell cortex localization of the I/LWEQ domain appeared to be dependent upon F-actin since the domain dissociated from the cortex after disruption of $\mathrm{F}$-actin in the presence of LatA. On the basis of our results, posterior localization of the I/LWEQ domain is unlikely to be related to the cGMP pathway, myosin II, or PI3K pathway. myosin // null cells and cells with a disrupted cGMP pathway showed posterior localization of the I/LWEQ domain as in wild-type cells. Further, PI3Ks and the products of PI3K PIP3 had no effect on localization of the domain during migration.

For a cell to migrate, F-actin is assembled at the front of cells, producing an extension toward chemoattractants through a series of F-actin binding proteins such as coronin, Arp2/3 complex, and actinin, which are recruited to the leading edge of cells (Kortholt and van Haastert, 2008; Lee and Jeon, 2012; Ridley et al., 2003). In our study, coronin, used as a marker for newly formed F-actin, accumulated at the front of cells along with the bottom as foci. The I/LWEQ domain in RapGAP3 was also found at foci at the bottom of cells, which suggests that, along with its F-actin dependent cell cortex localization, the domain belongs to a family of $\mathrm{F}$-actin binding proteins. However, the I/LWEQ domain was localized to the posterior but not anterior region, as with an F-actin binding protein.

To explain the result of posterior localization of the $1 / L W E Q$ domain as an F-actin binding protein in migrating cells, we propose that there might be two different types of F-actin with different binding affinities. One is newly formed F-actin filament at the front of cells while the other is pre-existing F-actin at the rear and lateral sides of cells. Our data suggest that the I/LWEQ domain may bind only to the pre-existing F-actin but not the newly formed F-actin at the front. Recent papers have reported a structural polymorphism in F-actin. F-actin is known to have a variable twist as well as a variable tilt of subunits (Galkin et al., 2010). It has been suggested that different actinbinding domains have different affinities for F-actin filaments in functionally distinct regions of the cytoskeleton, based on the studies about localization of the actin-binding domains of Filamin, which localizes to the rear of polarized cells, and actinin,
Fig. 4. Localization of the $I / L W E Q$ domain in mutants. The $I / L W E Q$ domain (GFP-137) was introduced into myosin /I null cells and two cGMP mutants, $g C A$ (guanylyl cyclase A)/sGC (soluble guanylate cyclase) null cells and cGMP-specific phosphodiesterase null cells, after which localization of the domain in migrating cells was analyzed. In addition, localization of the I/LWEQ domain in wild-type cells pretreated with the inhibitor LY294002 was also analyzed. which is enriched in new pseudopods and at the front of cells (Washington and Knecht, 2008). Myosin II motor domain localizes to the posterior of cells during migration (Jeon et al., 2007a; Kortholt and van Haastert, 2008; Lee and Jeon, 2012). It has been suggested that myosin II preferentially binds to stretched actin filaments in the rear cortex and cleavage furrows, and stretching of the actin filament itself increases its affinity for the myosin II motor domain (Kortholt and van Haastert, 2008; Uyeda et al., 2011). The I/LWEQ domain of RapGAP3 might have preferential binding affinity for stretched Factin in the rear cortex and lateral sides of cells as opposed to the cross-linked, newly formed F-actin in the front of cells, helping establish cell polarity by locally modulating Rap1 activity. At the leading edge, actin is assembled as a dendritic network forming a lamellipodial shape (Galkin et al., 2010; Ridley et al., 2003). In contrast, at the posterior region, F-actin is oriented parallel to the long axis of migration, which is consistent with the specific localization of the actin-binding proteins Spectrin (Medina et al., 2002; Wang et al., 1999), Talins (Tsujioka et al., 2012; Weber et al., 2002), myosin II (Jeon et al., 2007a; Meili et al., 2010; Uyeda et al., 2011), and the I/LWEQ domain of RapGAP3.

In conclusion, the polarity of cells is formed by diverse signaling molecules localized to the poles of cells in response to extracellular signals. Our results show that one type of protein, RapGAP3, has the ability to localize to either of the two poles. The I/LWEQ domain, which is known as an F-actin-binding region, in the central region of RapGAP3 was sufficient for posterior localization in migrating cells, whereas the GAP domain was required for anterior localization. The I/LWEQ domain appears to localize to the cell cortex in an F-actin dependent manner. However, it seems that the I/LWEQ domain was excluded by the newly formed $\mathrm{F}$-actin at protruding regions rather than binding to F-actin. These controversial results raise a possibility that there are two different types of F-actin with different binding affinities. We suggest that the I/LWEQ domain of RapGAP3 might bind only to preexisting F-actin at the lateral and posterior regions of the cell but not to newly formed F-actin at the leading edge. Additional studies would be helpful to determine whether or not the I/LWEQ domain binds to newly formed 
F-actin or preexisting F-actin. This study provides new insights into the posterior localization of signaling molecules in response to chemoattractant stimulation.

\section{ACKNOWLEDGMENTS}

This research was supported by Basic Science Research Program through the National Research Foundation of Korea (NRF) funded by the Ministry of Education, Science and Technology (2011-0022220), and by research funds from Chosun University to T.J. Jeon, 2011.

\section{REFERENCES}

Brett, T.J., Legendre-Guillemin, V., McPherson, P.S., and Fremont, D.H. (2006). Structural definition of the F-actin-binding THATCH domain from HIP1R. Nat. Struct. Mol. Biol. 13, 121-130.

Cha, I., and Jeon, T.J. (2011). Dynamic localization of the actinbundling protein cortexillin I during cell migration. Mol. Cells 32 281-287.

Cha, I., Lee, S.H., and Jeon, T.J. (2010). Chemoattractant-mediated Rap1 activation requires GPCR/G proteins. Mol. Cells 30, 563-567.

Galkin, V.E., Orlova, A., Schroder, G.F., and Egelman, E.H. (2010). Structural polymorphism in F-actin. Nat. Struct. Mol. Biol. 17, 1318-1323.

Jeon, T.J., Lee, D.J., Merlot, S., Weeks, G., and Firtel, R.A. (2007a). Rap1 controls cell adhesion and cell motility through the regulation of myosin II. J. Cell Biol. 176, 1021-1033.

Jeon, T.J., Lee, D.J., Lee, S., Weeks, G., and Firtel, R.A. (2007b). Regulation of Rap1 activity by RapGAP1 controls cell adhesion at the front of chemotaxing cells. J. Cell Biol. 179, 833-843.

Jeon, T.J., Lee, S., Weeks, G., and Firtel, R.A. (2009). Regulation of Dictyostelium morphogenesis by RapGAP3. Dev. Biol. 328, 210-220.

Kolsch, V., Charest, P.G., and Firtel, R.A. (2008). The regulation of cell motility and chemotaxis by phospholipid signaling. J. Cell Sci. 121, 551-559.

Kooistra, M.R., Dube, N., and Bos, J.L. (2007). Rap1: a key regulator in cell-cell junction formation. J. Cell Sci. 120,17-22.

Kortholt, A., and van Haastert, P.J. (2008). Highlighting the role of Ras and Rap during Dictyostelium chemotaxis. Cell. Signal. 20, 1415-1422.

Kortholt, A., Bolourani, P., Rehmann, H., Keizer-Gunnink, I., Weeks, G., Wittinghofer, A., and Van Haastert, P.J. (2010). A Rap/phosphatidylinositol 3-kinase pathway controls pseudopod formation [corrected]. Mol. Biol. Cell 21, 936-945.

Lee, M.R., and Jeon, T.J. (2012). Cell migration: regulation of cytoskeleton by Rap1 in Dictyostelium discoideum. J. Microbiol. $50,555-561$.

McCann, R.O., and Craig, S.W. (1997). The I/LWEQ module: a conserved sequence that signifies F-actin binding in functionally diverse proteins from yeast to mammals. Proc. Natl. Acad. Sci. USA 94, 5679-5684.

Medina, E., Williams, J., Klipfell, E., Zarnescu, D., Thomas, G., and Le Bivic, A. (2002). Crumbs interacts with moesin and beta(Heavy)spectrin in the apical membrane skeleton of Drosophila. J. Cell Biol. 158, 941-951.

Meili, R., Alonso-Latorre, B., del Alamo, J.C., Firtel, R.A., and Lasheras, J.C. (2010). Myosin II is essential for the spatiotemporal organization of traction forces during cell motility. Mol. Biol. Cell 21, 405-417.

Mun, H., and Jeon, T.J. (2012). Regulation of actin cytoskeleton by Rap1 binding to RacGEF1. Mol. Cells 34, 71-76.

Parkinson, K., Bolourani, P., Traynor, D., Aldren, N.L., Kay, R.R., Weeks, G., and Thompson, C.R. (2009). Regulation of Rap1 activity is required for differential adhesion, cell-type patterning and morphogenesis in Dictyostelium. J. Cell Sci. 122, 335-344.

Raaijmakers, J.H., and Bos, J.L. (2009). Specificity in Ras and Rap signaling. J. Biol. Chem. 284, 10995-10999.

Ridley, A.J., Schwartz, M.A., Burridge, K., Firtel, R.A., Ginsberg, M.H., Borisy, G., Parsons, J.T., and Horwitz, A.R. (2003). Cell migration: integrating signals from front to back. Science 302, 1704-1709.

Sanchez-Madrid, F., and Serrador, J.M. (2009). Bringing up the rear: defining the roles of the uropod. Nat. Rev. Mol. Cell Biol. 10, 353-359.

Sasaki, A.T., Chun, C., Takeda, K., and Firtel, R.A. (2004). Localized Ras signaling at the leading edge regulates PI3K, cell polarity, and directional cell movement. J. Cell Biol. 167, 505-518.

Senetar, M.A., Foster, S.J., and McCann, R.O. (2004). Intrasteric inhibition mediates the interaction of the I/LWEQ module proteins Talin1, Talin2, Hip1, and Hip12 with actin. Biochemistry 43 , 15418-15428.

Tsujioka, M., Yumura, S., Inouye, K., Patel, H., Ueda, M., and Yonemura, S. (2012). Talin couples the actomyosin cortex to the plasma membrane during rear retraction and cytokinesis. Proc. Natl. Acad. Sci. USA 109, 12992-12997.

Uyeda, T.Q., Iwadate, Y., Umeki, N., Nagasaki, A., and Yumura, S. (2011). Stretching actin filaments within cells enhances their affinity for the myosin II motor domain. PLoS One 6, e26200.

Wang, X.Y., Ostberg, J.R., and Repasky, E.A. (1999). Effect of feverlike whole-body hyperthermia on lymphocyte spectrin distribution, protein kinase $\mathrm{C}$ activity, and uropod formation. J. Immunol. 162, 3378-3387.

Washington, R.W., and Knecht, D.A. (2008). Actin binding domains direct actin-binding proteins to different cytoskeletal locations. BMC Cell Biol. 9, 10.

Weber, I., Niewohner, J., Du, A., Rohrig, U., and Gerisch, G. (2002). A talin fragment as an actin trap visualizing actin flow in chemotaxis, endocytosis, and cytokinesis. Cell Motil Cytoskeleton 53, 136-149. 